\title{
Effect of inbreeding and heritability of sperm competition success in the bulb mite Rhizoglyphus robini
}

\author{
M Konior ${ }^{1,2}$, L Keller ${ }^{2}$ and J Radwan ${ }^{1}$ \\ ${ }^{1}$ Institute of Environmental Sciences, Jagiellonian University, ul. Gronostajowa 7, 30-387 Cracow, Poland; ' ${ }^{2}$ Department of Ecology and \\ Evolution, BB, University of Lausanne, Switzerland
}

\begin{abstract}
Sperm competition is a potent evolutionary force shaping the reproductive biology of most animal species. Here, we estimated the heritability of sperm competition success in the promiscuous bulb mite Rhizoglyphus robini. Sperm competition success was measured with the sterile male technique as the proportion of eggs fertilised by the second of three males mated with a single female. Sperm competition success responded significantly to selection. The heritability estimated from the response to five generations of selection was 0.13 . We also estimated the effect of
\end{abstract}

inbreeding on sperm competition success. Males produced by sib-mating $(F=0.25)$ had a significantly lower sperm competition success than outbred males. The estimated coefficient of inbreeding depression was 0.53. Such high inbreeding depression together with moderately low heritability is consistent with the view that sperm competitive ability is under strong directional selection and strongly influences the reproductive success of males.

Heredity (2005) 94, 577-581. doi:10.1038/sj.hdy.6800649

Published online 2 March 2005

Keywords: sexual selection; quantitative genetics; dominance; additive genetic variation

\section{Introduction}

In many animal species, females mate with several males (Birkhead and Møller, 1998; Simmons, 2001), thus creating the opportunity for sperm competition (Parker, 1970). Consequently, male success in sperm competition can be a major determinant of male reproductive success, and traits affecting sperm competitiveness can be expected to be under strong directional selection. Such traits include sperm number (eg, Møller, 1989), spermoffensive traits, such as modified genitalia or seminal fluids that allow removal or incapacitation of sperm inseminated earlier (eg, Waage, 1979; Radwan and Witaliński, 1991; Clark et al, 1995), and traits such as copulatory plugs that protect own sperm from manipulation by other males (eg, Devine, 1975; Birkhead and Møller, 1998; Simmons, 2001).

Traits under strong directional selection tend to have low heritabilities (Mousseau and Roff, 1987), but hypotheses concerning evolution of mating systems often assume substantial heritability in sperm competition success. For example, Keller and Reeve (1995) pointed out that multiple-mating by females may rapidly spread when initially a small fraction of the females mate multiply and if there is a heritable difference among males in sperm competitive success. Similarly, Yasui (1997) formally showed that if sperm competitive success correlates genetically with viability, multiple-mating will

Correspondence: Dr J Radwan, Institute of Environmental Sciences, Jagiellonian University, ul. Gronostajowa 7, 30-387 Cracow, Poland.

E-mail:radwan@eko.uj.edu.pl

Received 26 August 2004; accepted 12 January 2005; published online 2 March 2005 be favoured because of the resulting increase in fitness of the progeny.

Despite the evolutionary importance of sperm competition (reviewed in Birkhead and Møller, 1998; Simmons, 2001), little is known about the genetic components underlying variation in sperm competition success. Clarke et al (1995) found that a polymorphism at four accessory gland protein genes is associated with the ability of Drosophila melanogaster males to resist sperm displacement. However, a later study failed to find a significant additive genetic variance for any component of sperm competition success (Hughes, 1997). At least two reasons may account for this pattern. The first is a female $\times$ male interaction such that a male with a given genetic background may outcompete a male with another genetic background with some females, but not with females of another genetic background. Pairwise tests among six isogenetic lines of D. melanogaster indeed revealed that the success of a particular male is dependent on the genotype of the female with which he mates (Clark and Begun, 1998). The second reason is a male $\times$ male interaction with the sperm competitive ability of a given male genotype depending on the genotype of the competing male (Keller and Reeve, 1995; Prout and Bundgaard, 1977). Experimental evidence also suggest such a male $\times$ male interaction occurs as the relative success of six chromosome-extracted lines was found to depend on the genetic background of the competing males (Clark et al, 1999).

The lack of additive genetic variance for sperm competition success in D. melanogaster contrasts with the results obtained in the bulb mite Rhizoglyphus robini. In this species, Radwan (1998) found a significant association between father and son success in sperm 
competition with a narrow-sense heritability of $0.28 \pm 0.16$ (Mean \pm s.e.). The heritability value for sperm competition success observed in the bulb mite is within the range of heritability values reported for life-history traits presumably under strong directional selection (Mousseau and Roff, 1987). Traits under strong directional selection should show a high degree of directional dominance because mutations will tend to decrease the trait value and nonrecessive mutation thus be rapidly eliminated (Lynch and Walsh, 1998, p 270). Consequently, traits under strong directional selection should also suffer strong inbreeding depression (Charlesworth and Charlesworth, 1999). To date the effect of inbreeding on sperm competition success has been measured only in $D$. melanogaster, where there is apparently substantial dominance variance and a significant negative effect of inbreeding on male sperm competitive ability (Hughes, 1997).

The aim of this study was two-fold. Firstly, as heritability characterises populations rather than species, we wanted to confirm that heritability of sperm competition success might be a general feature in the bulb mite Rhizoglyphus robini by studying another population from the one studied by Radwan (1998). Secondly, we wanted to determine whether inbreeding is also associated with negative effect on the sperm competition success of males of this species. Bulb mites copulate several times per day, resulting in intense sperm competition (Radwan and Siva-Jothy, 1996). The sperm from consecutive male partners mix to some degree in the spermatheca, but the last male to mate with the female tends to father more offspring (Radwan, 1997).

\section{Methods}

Bulb mites are diploid, sexually reproducing pests of subterranean structures of plants and foods in storage (reviewed in Diaz et al, 2000). The sex ratio of adults at the time of emergence is close to 1 (Gerson et al, 1983; personal observations). The mites used in this study came from a stock culture derived from a colony of about 200 individuals found on onions in a garden near Cracow, Poland in 1998. The culture was maintained as a large population ( $>1000$ individuals subdivided into six sub-populations mixed once a month) for about 50 generations. The subpopulations were kept in $2.5 \mathrm{~cm}$ diameter jars ( $2 \mathrm{~cm}$ high) maintained at $22-26^{\circ} \mathrm{C}$ and $>90 \%$ humidity. Individuals were fed ad libitum weekly with a 3:1 mixture of powdered yeast and wheat germ. Once a month, about $1 / 4$ of the food and debris containing several hundred mites at different stages of development were transferred to fresh jars. The same feeding, humidity and temperature conditions were also maintained throughout all the experiments described below. Individually isolated mites, as well as pairs and families of mites, were kept in $0.8 \mathrm{~cm}$ diameter glass tubes $(2 \mathrm{~cm}$ high) with Plaster of Paris bases soaked with water. The tubes were plugged with nonabsorbent cotton-wool.

\section{Measurement of sperm competition success}

The same procedure was used to determine sperm competition success in all the experiments performed in this study. To obtain virgin males and females for the sperm competition and inbreeding experiments, we isolated first nymphal instars (protonymph) and reared them individually to adulthood in $0.8 \mathrm{~cm}$ diameter tubes. Individuals were used in experiments 2-4 days after emergence.

Sperm competition success was investigated using the sterile male technique (Parker, 1970). Experimental males were tested in a triple-mating experiment. Virgin females were presented with three males in turn, each male for $2 \mathrm{~h}$ : first, an irradiated male, next the experimental male and finally another irradiated male. Two hours is enough for the majority of pairs to mate (in a similar setting, Radwan et al (2005) recorded about $90 \%$ pairs initiating copulation within $90 \mathrm{~min}, n=60$ ), but repeated mating within such short time is very rare. Thus, most pairs copulated once. After the last male was removed, females were left to lay eggs for 1 week.

To sterilise males, we irradiated them with a $20 \mathrm{krad}$ from a $\mathrm{Co}^{60}$ source. Such a dose prevents eggs fertilised by irradiated males from hatching without compromising sperm competitive ability of males (Radwan, 1997), and the proportion of eggs fertilised by the second, nonirradiated males $\left(\mathrm{P}_{2}\right)$ could be estimated from the proportion of hatched eggs. All sterilised males were taken from the stock population. Prior to irradiation, they were stored in groups of 100 males and 100 females in $2.5 \mathrm{~cm}$ diameter plastic jars for $12 \mathrm{~h}$. Simultaneously, experimental males, isolated in a $0.8-\mathrm{cm}$ diameter cells, were supplied with one female for the same period of time. We kept males with a female because they always have access to females under natural conditions. Keeping all experimental males with one female also allowed us to standardise their sperm reserves (Radwan, 1997) before the experiment.

\section{Selection experiment}

We mass-selected three lines for increased sperm competitiveness while simultaneously maintaining three independent control lines. In each selection line we measured sperm competitiveness of 100 males in the same manner as described above. The top 25 males were then mated with a virgin female randomly taken from the same selection line. After the progeny matured, four adult males were randomly selected from each family to obtain the next generation of 100 males whose sperm competition success was again measured. Pairings were random except that matings among full-sibs were avoided. The breeding scheme was identical in the control lines, with the difference that we randomly selected 25 males during each generation.

In the fifth generation, sperm competition success of males from selected lines was compared with that of males from control lines. Sperm competition success of 30 randomly selected males from each selected line and each control line was measured as described above. As only males were selected, the realised heritabilities for each selection line were calculated as twice the ratio of total response to cumulative selection differential (Falconer, 1989; Roff, 1997).

\section{Inbreeding depression}

To obtain virgin males and females, we isolated protonymphs collected from the stock population. Virgin females were mated with a randomly selected virgin male to obtain 60 full-sib families. For each family we 
isolated four protonymphs and mated the virgin females so obtained with either a brother or an unrelated male. In this way, we obtained 24 inbred and 25 outbred families. In each family we isolated 10 protonymphs to subsequently measure sperm competition success of the males (2-6 males per family). We used family means in statistical analyses to measure the effect of inbreeding.

\section{Statistics}

To test the significance of the response to selection, we used means of selected and control lines; normality of the distribution of the means can be assumed based on the central limit theorem because means estimated from large samples will be normally distributed irrespective of the shape of the population distribution (Mendenhall, 1983). As both the direction of the difference between selected and unselected lines and inbred and outbred lines can be predicted a priori (ie, higher $\mathrm{P}_{2}$ for selected lines and for outbreds), we used directed tests (Rice and Gaines, 1994). Directional tests are a compromise between one- and two-tailed tests that increase statistical power relative to the latter, but still allow rejection of hypotheses in the unanticipated direction. Following the convention proposed by Rice and Gaines (1994), $80 \%$ of the rejection region was in the anticipated direction, and $20 \%$ in unanticipated direction (as opposed to $50 \%$ in both directions in a two-tailed test).

Inbreeding depression was measured as $1-\hat{w}_{\mathrm{i}} / \hat{w}_{0}$, where $\hat{w}_{\mathrm{i}}$ and $\hat{w}_{\mathrm{o}}$ are means for inbred and outbred families. Significance of inbreeding depression was tested with $t$-statistic calculated as $t=\left[\hat{w}_{\mathrm{o}}-\hat{w}_{\mathrm{i}}\right] /\left(S D_{\mathrm{o}}(2 / L)^{1 / 2}\right)$, where $\mathrm{SD}_{\mathrm{o}}$ is the standard deviation of the trait measured in outbred families and $L$, the number of families, with L-1 degrees of freedom (Lynch and Walsh, 1998). Family mean $P_{2}$ values were square-root transformed to achieve normality.

\section{Results}

After four generations of selection, the fertilisation success of males was significantly higher $\left(t_{4}=2.59\right.$; $\left.P_{\text {DIR }}=0.046\right)$ in selected lines than in control lines (Figure 1). The estimated heritabilities in the three selected lines were $0.025,0.102$ and 0.313 , giving an average $( \pm$ s.e.) estimate of $0.130( \pm 0.098)$.

The estimated proportion of offspring fathered was significantly lower for inbred males than non-inbred males $\left(t_{24}=4.952, P_{\text {DIR }}<0.001\right)$. Overall, the proportion of offspring fathered by inbred males was 0.205

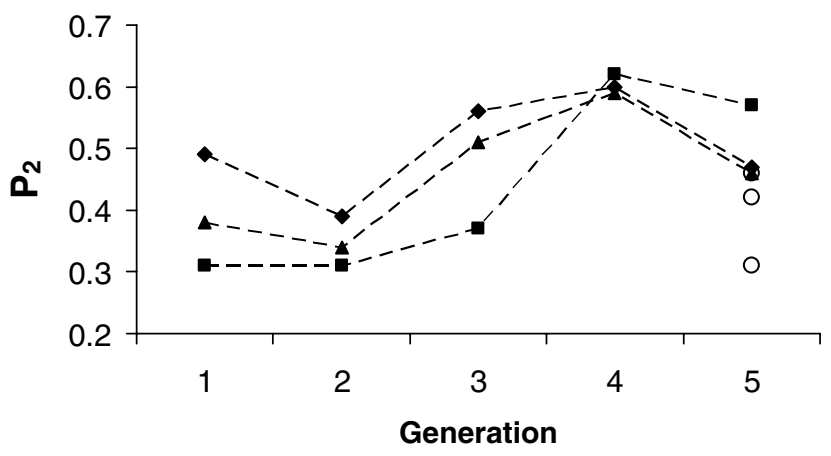

Figure 1 The response to selection on increased success in sperm competition. Filled symbols: selected lines, open symbols: controls. (s.d. $=0.145)$ compared to 0.444 (s.d. $=0.175)$ for outbred males. Thus, the estimated inbreeding depression was 0.53 for an inbreeding coefficient of $F=0.25$.

\section{Discussion}

The first important finding of this study is that there is a significant heritability for sperm competitive ability in bulb mites. The estimated heritability was 0.130 (s.e. \pm 0.098$)$. This value is slightly smaller than the value previously reported for another population of the same species (0.284 s.e. \pm 0.160 , Radwan, 1998), but the standard errors of the two estimates overlap. The heritability of sperm competition success in $R$. robini is close to the average values measured for life-history traits in D. melanogaster (0.12; Roff and Mousseau, 1987) and other animals (0.26; Mousseau and Roff, 1987), but lower than the average heritability of morphological traits (0.5), which are thought to be less associated with fitness than life-history traits (Mousseau and Roff, 1987; Roff and Mousseau, 1987).

The heritability of sperm competition has been quantified in only very few other species. Hughes (1997) failed to detect a significant additive genetic variance for male sperm competitiveness in D. melanogaster despite the fact that polymorphic alleles affecting sperm precedence were shown to occur in natural populations (Clark et al, 1995). However, as discussed in the introduction, this pattern can be explained by nonadditive effects of genes affecting sperm competitiveness. In the field cricket Teleogryllus oceanicus, Simmons (2003) also failed to find a significant sire effect on sperm competitiveness. There was, however, a significant dam effect, suggesting either a X-linked inheritance or a maternal effect (Simmons, 2003).

Although two out of the three studies failed to find a heritable component to sperm competitiveness, some indirect evidence for heritability of sperm competitiveness has been obtained in some other species (see Keller and Reeve, 1995; Pizzari and Birkhead, 2002). For example, Simmons and Kotiaho (2002) reported significant heritabilities of traits associated with sperm competition success in the dung beetle Onhtophagus taurus, while Bernasconi and Keller (2001) found that sons of polyandrous females of the flour beetle Tribolium castaneum had improved offensive component of sperm competitiveness compared to males produced by females that mated with only one male. In the domestic fowl, Froman et al (2002) also reported a heritability of 0.30 for sperm motility, a character that strongly influences fertilisation efficiency. Several other studies have shown that mating with several males affected components of fitness of offspring (eg Evans and Magurran, 2000; Konior et al, 2001; Sakaluk et al, 2002), suggesting a genetic correlation between sperm competitiveness and fitness traits, and hence some degree of additive genetic variance for sperm competitiveness. However, such results of multi-male mating experiments should be considered with some care because they can be associated with nongenetic effects such as maternal effects (Tregenza et al, 2003; Kozielska et al, 2004). Thus, results of experiments comparing the progeny of singly and multiply mated females do not allow us to draw a firm conclusion on the heritability of sperm competitiveness. For example, in spite of the heritability of sperm 
competitiveness in the bulb mite, Konior (2004) found no significant difference in sperm competitiveness between sons of polyandrous and monandrous females, possibly because multi-male mating produces negative maternal effects on sons, but not on daughters (Kozielska et al, 2004).

The second important finding of this study was that inbreeding greatly affected the competitive ability of males. The estimated effect was $53 \%$ for a value of inbreeding of $F=0.25$. Studies in other species showed that the average effect of a similar level of inbreeding on life-history traits that presumably are under strong directional selection was $11.8 \%$ (DeRose and Roff, 1999), while the effect was much lower (2.2\%) for weakly selected morphological traits. In $R$. robini, inbreeding depression in female fecundity after full-sib mating was estimated at 22\% (Radwan, 2003). Thus, the high inbreeding depression on sperm competition success of bulb mites suggests that this trait shows strong directional dominance, a likely result of its close association with fitness (Lynch and Walsh, 1998). Indeed, inbreeding depression estimates of this order of magnitude are commonly found for traits strongly affecting fitness (eg, Table 8.9 in Roff, 1997).

To our knowledge, the effect of inbreeding on sperm competitive success has been directly estimated in only one other species, D. melanogaster (Hughes, 1997). Males made homozygous for the third chromosome had 25 and $33 \%$ lower sperm precedence when mating as first or second male, respectively. However, indirect evidence also suggests an effect of inbreeding in mammals, with inbred males showing lower sperm motility as well as other ejaculate characteristics presumably decreasing sperm competitiveness (Gomendio et al (2000) and references therein).

In our experiments, sperm competition success was assessed in a situation where males could both displace (or incapacitate) the sperm of a previous male, and defend their sperm from displacement by a male that mated subsequently. This is probably the most common situation in this promiscuous species, with the effect that male reproductive success depends on the net effect of both sperm defence and offence. However, as documented in fruit flies (Clark et al, 1995) and flour beetles (Bernasconi and Keller, 2001), the offensive and defensive aspects of sperm competitive ability can be uncorrelated. Therefore, it would be interesting in future studies to analyse separately the quantitative genetics of these two components.

\section{Acknowledgements}

The project was funded by the Polish State Committee for Scientific Research KBN 0408/P04/2001/21 to JR. MK was funded by the Commission Federale des Bourses pour Etudiants Etrangers and LK by several grants from the Swiss NSF. We thank Karen Parker for reading the previous version of this manuscript, and two anonymous referees for their comments.

\section{References}

Bernasconi G, Keller L (2001). Female polyandry affects their sons' reproductive success in the red flour beetle Tribolium castaneum. J Evol Biol 14: 186-193.
Birkhead TR, Møller AP (1998). Sperm Competition and Sexual Selection. Academic Press: London.

Charlesworth B, Charlesworth D (1999). The genetic basis of inbreeding depression. Genet Res Camb 74: 329-340.

Clark AG, Aguade M, Prout T, Harshman LG, Langley $\mathrm{CH}$ (1995). Variation in sperm displacement and its association with accessory gland protein loci in Drosophila melanogaster. Genetics 139: 189-201.

Clark AG, Begun DJ (1998). Female genotypes affect sperm displacement in Drosophila. Genetics 149: 1487-1493.

Clark AG, Begun DJ, Prout T (1999). Female $\times$ male interactions in Drosophila sperm competition. Science 283: 217-220.

DeRose MA, Roff DA (1999). A comparison of inbreeding depression in life-history and morphological traits in animals. Evolution 53: 1288-1292.

Devine MC (1975). Copulatory plug in snakes: enforced chasity. Science 187: 844-845.

Diaz A, Okabe K, Eckenrode CJ, Villani MG, Oconnor BM (2000). Biology, ecology and management of the bulb mites of the genus Rhizoglyphus (Acari: Acaridae). Exp Appl Acarol 24: 85-113.

Evans JP, Magurran AE (2000). Multiple benefits of multiple mating in guppies. Proc Natl Acad Sci USA 97: 10074-10076.

Falconer DS (1989). Introduction to Quantitative Genetics, 3rd edn. Longman: Harlow.

Froman DP, Pizzari T, Feltmann AJ, Castillo-Juarez H, Birkhead TR (2002). Sperm mobility: mechanisms of fertilizing efficiency, genetic variation and phenotypic relationship with male status in the domestic fowl, Gallus gallus domesticus. Proc R Soc London B 269: 607-612.

Gerson U, Capua S, Thorens D (1983). Life history and life tables of Rhizoglyphus robini Claparede (Acari: Astigmata: Acaridae). Acarologia 24: 439-448.

Gomendio M, Casinello J, Roldan ERS (2000). A comparative study of ejaculate traits in three endangered ungulates with different levels of inbreeding: fluctuating asymmetry as an indicator of reproductive and genetic stress. Proc $R$ Soc London B 267: 875-882.

Hughes KA (1997). Quantitative genetics of sperm precedence in Drosophila melanogaster. Genetics 145: 139-151.

Keller L, Reeve HK (1995). Why do females mate with multiple males? The sexually selected sperm hypothesis. Adv Stud Behav 24: 291-315.

Konior M (2004). Koszty i zyski z poliandrii u Rhizoglyphus robini (Clap) Acari, Acaridae. PhD Thesis, Jagiellonian University.

Konior M, Radwan J, Kołodziejczyk M (2001). Polyandry increases offspring fecundity in the bulb mite. Evolution 55: 1893-1896.

Kozielska M, Krzemiñska A, Radwan J (2004). Good genes and the maternal effects of polyandry on offspring reproductive success in the bulb mite. Proc R Soc London B 271: 165-170.

Lynch M, Walsh B (1998). Genetics and Analysis of Quantitative Traits. Sinauer Associates: Sunderland, MA.

Mendenhall W (1983). Introduction to Probability and Statistics. Duxbury Press: Boston.

Møller AP (1989). Ejaculate quality, testes size and sperm production in mammals. Funct Ecol 3: 91-96.

Mousseau TA, Roff DA (1987). Natural selection and the heritability of fitness components. Heredity 59: 181-197.

Parker GA (1970). Sperm competition and its evolutionary effect on copula duration in the fly Scatophaga stercoraria. J Insect Physiol 16: 1301-1328.

Pizzari T, Birkhead TR (2002). The sexually-seelcted sperm hypothesis: sex-biased inheritance and sexual antagonism. Biol Rev 77: 183-209.

Prout T, Bundgaard J (1977). The population genetics of sperm displacement. Genetics 85: 95-124.

Radwan J (1997). Sperm precedence in the bulb mite, Rhizoglyphus robini: context-dependent variation. Ethol Ecol Evol 9: 373-383. 
Radwan J (1998). Heritability of sperm competition success in the bulb mite, Rhizoglyphus robini. J Evol Biol 11: 321-327.

Radwan J (2003). Inbreeding depression in fecundity and inbred line extinction in the bulb mite, Rhizoglyphus robini. Heredity 90: 371-376.

Radwan J, Michalczyk Ł, Prokop Z (2005). Age-dependence of male mating ability and sperm competition success in the bulb mite. Anim Behav (in press).

Radwan J, Siva-Jothy MT (1996). The function of postinsemination mate association in the bulb mite Rhizoglyphus robini. Anim Behav 52: 651-657.

Radwan J, Witaliński W (1991). Sperm competition. Nature 352: 671-672.

Rice WR, Gaines SD (1994). ‘Heads I win, tails you lose': testing directional alternative hypotheses in ecological and evolutionary research. Trends Ecol Evol 9: 235-237.

Roff DA (1997). Evolutionary Quantitative Genetics. Chapman \& Hall: New York.

Roff DA, Mousseau TA (1987). Quantitative genetics and fitness: lessons from Drosophila. Heredity 58: 103-118.
Sakaluk SK, Schaus JM, Eggert AK (2002). Polyandry and fitness of offspring reared under varying nutritional stress in decorated crickets. Evolution 56: 1999-2007.

Simmons LW (2001). Sperm Competition and its Evolutionary Consequences in the Insects. Princeton University Press: Princeton.

Simmons LW (2003). The evolution of polyandry: patterns of genotypic variation in female mating frequency, male fertilization success and a test of the sexy-sperm hypothesis. J Evol Biol 16: 624-634.

Simmons LW, Kotiaho JS (2002). Evolution of ejaculates: patterns of phenotypic and genotypic variation and condition dependence in sperm competition traits. Evolution 56: 1622-1631.

Tregenza T, Wedell N, Hosken DJ, Ward PI (2003). Maternal effects on offspring depend on female mating pattern and offspring environment in yellow dung flies. Evolution 57: 297-304.

Waage JK (1979). Dual function of the damselfly penis: sperm removal and transfer. Science 203: 916-918.

Yasui Y (1997). A 'good-sperm' model can explain the evolution of costly multiple mating by females. Am Nat 149: 573-583. 